\title{
LA REPÚBLICA DE GÉNOVA Y LA MONARQUÍA HISPÁNICA (SIGLOS XVI-XVII)
}

\author{
INTRODUCCIÓN
}

El presente monográfico aborda el estudio de las estrechas relaciones bilaterales entabladas entre la Monarquía Hispánica y la república de Génova a partir de la firma del acuerdo de condotta entre Carlos V y Andrea Doria en 1528 y hasta la desmembración del imperio español en Europa con la muerte del último representante de la dinastía Habsburgo en 1700. Un periodo que, en palabras de Costantini, se caracterizaría por una verdadera simbiosis y por un proceso de condicionamiento recíproco entre ambos modelos cuya fuerte interdependencia nos induciría a hablar, de manera más correcta, de un sistema imperial hispano-genovés ${ }^{1}$.

Estaríamos, por lo tanto, en presencia de un imponente conglomerado de poder en el que ambos socios cubrieron con eficacia, y en un panorama de estabilidad social y política desconocido en el resto de Europa, una serie de funciones que les permitieron ejercer una posición hegemónica en el continente hasta bien entrado el siglo XVII. La Monarquía Hispánica fue la encargada de proveer al conjunto de la pertinente protección militar y de amplias posibilidades de promoción para sus elites gracias al acceso privilegiado a sus ricos mercados y a una imponente capacidad de patronazgo regio. Por su parte, la república proporcionó al sistema el crédito y los capitales necesarios para sostener el

1 Costantini, C.: La Repubblica di Genova nell età moderna, Turín, 1978. Es, sin duda, una de las mejores síntesis sobre Génova en la Edad Moderna. Recientemente ha aparecido un compendio general de la Historia de Génova en el que dos de los mejores conocedores de las relaciones de la república con la Monarquía Hispánica se encargan de los capítulos reservados a la Edad Moderna. El estudio de Arturo Pacini sobre el siglo XVI y de Carlo Bitossi sobre los siglos XVII y XVIII incorporan un balance crítico con las principales aportaciones bibliográficas, PUNCUH, D. (ed.): Storia di Genova. Mediterraneo, Europa, Atlantico, Génova, 2003, pp. 325-508. 
agotador esfuerzo militar y suministró una serie de recursos navales fundamentales para establecer una adecuada comunicación entre los dispersos territorios de la Monarquía. Génova, a pesar de no formar parte de los dominios patrimoniales de los Habsburgo y de gozar de una relativa autonomía política, acabó por convertirse en uno de los principales vértices geoestratégicos del sistema imperial hispánico y se constituyó en una pieza preciosa para desquilibrar la balanza del lado español en su enfrentamiento secular con la dinastía francesa. En calidad de puerto natural del ducado de Milán y de nexo articulador entre los dominios italianos e ibéricos de la corona, a partir de la rebelión de Flandes, se convertiría también en el primer eslabón del Camino Español y en el mejor lugar de encuentro y difusión de un modelo cultural híbrido, de marcado carácter internacional, en el que se fundían elementos procedentes de todos los dominios de la Monarquía².

Un estudio pormenorizado sobre la naturaleza de los lazos de complementariedad entre ambos aliados y en torno al alcance de tan intensa colaboración nos permitirá ofrecer un cuadro mejor articulado de las relaciones entre las distintas potencias europeas, así como de las transformaciones sociales y económicas derivadas de las cambiantes relaciones bilaterales entre las dos piezas de este mismo engranaje. Las posibilidades de estudio son muy vastas y cuentan además con la existencia de un rico y abundante material archivístico que, en la mayoría de los casos, sigue estando inédito. No nos referimos tan sólo a los bien catalogados fondos de Estado del Archivo General de Simancas ${ }^{3}$, ni al abundante material conservado en la sección Archivio Segreto del Archivio di Stato di Genova. Las múltiples facetas de las relaciones hispano-genovesas permiten encontrar infinitos filones documentales en la práctica totalidad de las secciones de ambos archivos, por no mencionar los interesantes materiales almacenados en la Biblioteca Nacional de Madrid o en el Archivio Storico del Comune di Genova así como en los archivos de protocolos italianos y españoles, habida cuenta de la dilata implantación de las comunidades genovesas en las principales ciudades de la Monarquía. Dispersión territorial que hace recomendable una visita a los fondos documentales napolitanos, sicilianos o belgas para poder obtener una verdadera visión de conjunto sobre tan complejos vínculos.

Vistas las posibilidades de trabajo y la abundancia de los materiales disponibles sorprende observar la pobreza de las aportaciones dedicados al estudio de

\footnotetext{
2 Para un desarrollo más extenso sobre determinadas cuestiones que planteamos en esta introducción puede consultarse el reciente balance que hemos realizado sobre las características del entramado hispano-genovés, HERRERO SÁNCHEZ, M.: «Génova y el sistema imperial hispánico» en Álvarez-Ossorio, A. y García García, B. (eds.), La Monarquía de las Naciones. Patria, nación y naturaleza en la Monarquía de España, Madrid, 2004, pp. 528-562.

3 Magdaleno, R.: Papeles de Estado Génova (siglos XVI-XVIII). Catálogo XXV del Archivo General de Simancas, Valladolid, 1971. Sobre las posibilidades que ofrecen estos fondos para el estudio de la situación interior de la república véase la reseña que de dicho catálogo realizó Edoardo Grendi para Rivista Storica Italiana, LXXXIV, 4, (1972), pp. 1125-1128.
}

Hispania, LXV/1, núm. 219 (2005) 9-20 
las relaciones entre la Monarquía Hispánica y Génova. Más aún si tenemos en cuenta la revalorización efectuada en los últimos años sobre el papel ejercido por las repúblicas mercantiles, que se nos describen como una excepción modernizador en una Europa dominada por los sistemas dinástico-señoriales ${ }^{4}$. $\mathrm{Cu}$ riosamente, este deseo de acentuar la originalidad del modelo republicano viene a clarificar, en parte, el escaso interés historiográfico suscitado por el caso genovés, en especial si se compara con las innumerables contribuciones relativas a las Provincias Unidas o a Venecia. La escasa autonomía política de la república y los fuertes lazos financieros que la ligaban a la Monarquía Hispánica, contrapunto de la modernidad y paradigma de la intervención arbitraria del estado en la economía ${ }^{5}$, parecen converger con los argumentos ya expuestos por la tradición romántico-liberal decimonónica a la hora de valorar el peso de Génova en la Europa. Según estos planteamientos, el acuerdo de Andrea Doria con el Emperador sería una de las más palpables expresiones del inicio de la dominación extranjera sobre Italia e impulsaría un proceso de oligarquización de los dinámicos grupos mercantiles de la república que habían protagonizado la expansión naval genovesa durante la Edad Media ${ }^{6}$. Una visión idealizada del pasado medieval que, como se ve en los trabajos de Pacini, Kirk y Herrero en el presente monográfico, se puede encontrar ya entre aquellos tratadistas políticos que se mostraron más críticos con los asentistas de galeras y los opulentos

4 No es el momento de señalar los límites de un cuadro interpretativo que deja de lado los fuertes elementos de interdependencia y los procesos de transformación mutua entre los modelos monárquicos y republicanos, cuestión que constituye el núcleo de nuestro actual proyecto de investigación. Un buen balance sobre los aspectos relativos al republicanismo, en el que Génova tiene reservado un papel secundario, es el de GeLDEREN, M. Van, SKInNER, Q. (Eds.): Republicanism. A Shared European Heritage, 2 Vols., Cambridge, 2002. Las primeras conclusiones a las que hemos llegado pueden seguirse en HERRERO SÁNCHEZ, M.: «Las repúblicas mercantiles, ¿Alternativa al modelo dinástico? Génova, las Provincias Unidas y la Monarquía Hispánica en la segunda mitad del siglo XVII», en Crespo, A. y Herrero, M. (eds.), España y las 17 Provincias de los Países Bajos. Una revisión historiográfica (siglos XVI-XVIII), Córdoba, 2002, 1 Vol., pp. 189-227.

5. Planteamientos expuesto de modo descarnado por Peter Burke para quien Castilla constituiría el clásico ejemplo de cultura anti-empresarial en la que los valores político-religiosos se impondrían sobre los meramente económicos, BURKE, P., «Republics of Merchants in Early Modern Europe.» en J. Baecheler, J. A. Hall y M. Mann (eds.), Europe and the Rise of Capitalism, Oxford, 1988, PP. 220-233. Un interesante redimensionamiento de todos estos estereotipos y de las visiones distorsionadas sobre la economía y la sociedad castellanas ofrecidas por Douglas North, Inmanuel Wallerstein o David Landes lo constituye la reciente síntesis de YUN CASALILla, B.: Marte contra Minerva. El precio del imperio español, c. 1450-1600, Barcelona, 2004.

6 Sobre esta valoración de la historiografía tradicional italiana véase, PACINI, A.: La Genova di Andrea Doria nell Impero di Carlo V, Florencia, 1999, pp. 9-14. Braudel subraya, por el contrario, los avances económicos aportados por Génova a la que define como «ville capitaliste par excellence», BRAUdel, F.: Civilisation matérielle, économie et capitalisme, XVe-XVIIIe siècles. III: Les Temps du monde. París, 1979, p. 140. Por su parte, Edoardo Grendi enfatiza el componente privado del estado genovés con un poder central extremadamente débil, una burocracia poco desarrollada y unas finanzas públicas dependientes del capital privado, GRENDI, E., Introduzione alla storia moderna della Repubblica di Genova, Génova, 1973. 
banqueros al servicio del rey Católico, a los que acusaban de ser los causantes de la crisis de la manufactura genovesa y de llevar un tren de vida aristocrático alejado de la sobriedad y la virtud republicanas ${ }^{7}$.

La historiografía española también parece haberse hecho eco del enfoque con el que fueron observados los genoveses por los contemporáneos. Sin recurrir a los planteamientos peyorativos y fuertemente críticos utilizados por los arbitristas o por determinados procuradores en Cortes, para los que los hombres de negocios de la república parecían ser los causantes de la ruina del reino debido a la masiva extracción de plata y a los elevados intereses de sus préstamos, la única faceta de las intensas relaciones hispano-genovesas que ha despertado un cierto interés hasta el momento es aquella relativa a las actividades financieras de los asentistas. La noción de «siglo de los genoveses» atribuida a Felipe Ruiz Martín y popularizada por Fernand Braudel, se ha convertido en un lugar común y ha dado pie a una dilatada producción historiográfica caracterizada por el rigor y la calidad de las aportaciones. Trabajos como los de $\mathrm{Ca}$ rande, Otte, Domínguez Ortiz, Doria, Neri, Kellebenz o Muto han puesto de manifiesto que resultaba imposible entender el funcionamiento de la Monarquía Católica sin tener en cuenta el papel determinante jugado por unos hombres de negocios que parecían mediatizarlo todo y de los que dependía la suerte del sistema imperial en su conjunto ${ }^{8}$.

En el presente monográfico contamos con dos aportaciones realizadas por los que, sin lugar a dudas, son los principales especialistas españoles en esta materia que nos ofrecen el fruto de sus más recientes investigaciones. Carlos

\footnotetext{
7 Sobre estas cuestiones veánse las aportaciones de BITOSSI, C.: Il governo dei magnifici. Patriziato e politica a Genova fra Cinque e Seicento, Génova, 1990, libro sobre el que está preparando una revisión actualizada y, del mismo autor, «Il tempo deli oligarchi. Note sulla storia politica genovese nella prima età moderna», Annali dell'Università di Ferrara. Sezione lettere, Nuova serie 4, (2003), pp. 121-160; COSTANTINI, C.: «Politica e storiografia: l'epoca dei grandi repubblichisti» en La letteratura ligure. La Repubblica aristocratica (1528-1797), Génova, 1992, Vol. II, pp. 93-135 o SAVELLI, R. "La pubblicistica politica genovese durante le guerre civili del 1575», Atti della Società Ligure di Storia Patria, XX, 2, (1980), pp. 82-105.

8 CARANDE, R.: Carlos V y sus banqueros, Barcelona, 1977; Doria, G.: «Consideraciones sobre las actividades de un "factor cambista» genovés al servicio de la corona española» en Otazu, E. (ed.), Dinero y crédito (siglos XVI-XIX),, Madrid, 1978, pp. 279-293; DomíngueZ OrTIZ, A.: Política y Hacienda de Felipe IV, Madrid, 1983 ( $1^{\text {a }}$ ed. 1960); las contribuciones de Doria, Muto y Otte, realizadas en MADDALENA, A. de y KellebenZ, $\mathrm{H}$. (eds.): La repubblica internazionale del denaro, Bolonia, 1986; NeRI, E.: Uomini d'affari e di governo tra Genova e Madrid (secoli XVI e XVII), Milán, 1989; RuIz MARTín, F.: Las finanzas de la Monarquía Hispánica en tiempos de Felipe IV (1621-1665), Madrid, 1990 y, del mismo autor, Pequeño capitalismo y gran capitalismo. Simón Ruiz y sus negocios en Florencia, Barcelona, 1992; OTTE, E.: «El imperio genovés, 1522-1556», en Banchi pubblici, banchi privati e monti di pietà nell'Europa preindustriale. Amministrazione, tecniche operative e ruoli economici. Atti della Società Ligure di Storia Patria, XXXI (1991), págs. 247-263; CANOSA, R.: Banchieri genovesi e sourani spagnoli tra Cinquecento e Seicento, Roma, 1998.
}

Hispania, LXV/1, núm. 219 (2005) 9-20 
Álvarez Nogal9, a partir de los escasamente utilizados fondos del Archivo Histórico de Protocolos de Madrid y desde una poco habitual perspectiva de historia de la empresa, aborda el estudio de las principales firmas genovesas que actuaron en Castilla durante la primera mitad del siglo XVII. A través de un detenido análisis de sus estrategias familiares y de las características comunes de sus compañías, enfatiza su calidad como proveedoras de fondos, que parecía hacerlos insustituibles, y su papel de interlocutoras entre la Real Hacienda y las casas matrices radicadas en Génova, sin las que no hubiera sido posible firmar asientos con la Corona. Desde su punto de vista, más que por los negativos efectos de las bancarrotas de 1607 y 1627 — que dejaron indemnes a las firmas más poderosas - sería la desaparición biológica, entre 1639 y 1644 , la que explicaría su retirada con el agravante de que, frente a lo ocurrido a principios del reinado de Felipe III cuando los banqueros más veteranos fueron sustituidos por nuevas firmas, para la década de 1640 la Monarquía Hispánica había dejado de ser atractiva y los capitales genoveses parecían encaminarse hacia otras plazas.

La crisis de 1640 constituye el ámbito de estudio del artículo de Carmen Sanz Ayán ${ }^{10}$. Gracias a un vaciado sistemático de los fondos de Contadurías Generales del Archivo General de Simancas, se procede a un estudio de las provisiones efectuadas no sólo en plata, como había sido lo habitual hasta ahora, sino también en vellón. Los detallados cuadros que ofrece, con los nombres y las consignaciones realizadas por los principales asentistas de la corona, evidencian cómo, entre 1639 y 1649, la capacidad crediticia de la Monarquía cayó en un $58 \%$ lo que nos puede ayudar a comprender el desplazamiento internacional sufrido por los Habsburgo y sus dificultades para acudir a los innumerables frentes militares abiertos. La retirada parcial de los genoveses a partir de 1640, y hasta la bancarrota de 1647, se pone en relación con el proceso de integración en las redes aristocráticas castellanas de los principales banqueros y su alineamiento a favor de la facción nobiliaria enfrentada a Olivares, lo que les inclinaba a no identificarse con los métodos financieros extraordinarios puestos en marcha por el valido.

El impacto de las relaciones entabladas entre el monarca Católico y los hombres de negocios genoveses sobre la posición internacional de la Monarquía y sobre las transformaciones sociales y económicas experimentadas en Castilla hacen necesario ampliar el enfoque de nuestro análisis hacia determinados aspectos que han quedado relegados a un segundo término. En este sentido conviene recordar la labor realizada por determinados historiadores italianos, en

9 Álvarez Nogal, C.: El crédito de la monarquía hispánica en el reinado de Felipe IV, Ávila, 1997 así como, del mismo autor, «I genovesi e la monarchia spagnola tra Cinque e Seicento» Atti della Società Ligure di Storia Patria, XLI, 2, (2001), pp. 107-123.

10 SANZ AYÁn, C.: Los banqueros de Carlos II, Valladolid, 1988 así como la reciente compilación reactualizada de buena parte de sus mejores artículos sobre cuestiones financieras, SANZ AYÁN, C.: Estado, monarquía y finanzas. Estudios de Historia financiera en tiempos de los Austrias, Madrid, 2004. 
especial por Carlo Bitossi y Arturo Pacini, en su deseo de llevar a cabo un redimensionamiento de las perspectivas utilizadas hasta ahora para el estudio de la historia de Génova. Desde su punto de vista, los enfoques en apariencia contrapuestos entre los historiadores económicos, interesados en la dimensión internacional de la república, y los que, como Edoardo Grendi y Osvaldo Raggio $^{11}$, han apostado por un análisis local desde una perspectiva microhistórica deberían conciliarse para poder ubicar los procesos de cambio experimentados en el interior de la república en su necesario contexto europeo ${ }^{12}$. De este manera, podríamos observar los múltiples elementos de articulación y dependencia que se establecieron entre la Monarquía Hispánica y la república de Génova y el impacto que la evolución de las relaciones bilaterales ejerció en el desarrollo interno de ambos aliados. Salvo el trabajo de Arturo Pacini en torno al periodo de Andrea Doria ${ }^{13}$, no contamos en la actualidad con ningún otro estudio que elabore un panorama general de las relaciones hispano-genovesas durante el gobierno de los Austrias. El objetivo propuesto en las tres contribuciones restantes es el de ofrecer un primer acercamiento sobre estas cuestiones donde el lector podrá encontrar las necesarias referencias bibliográficas y las cuestiones de fondo que marcaron la evolución de dichas relaciones bilaterales a lo largo de los siglos XVI y XVII.

El artículo de Arturo Pacini analiza, a partir de un adecuado contraste entre las fuentes genovesas y la documentación de Simancas, el periodo en el que se asientan los fundamentos sobre los que reposará la alianza hispanogenovesa. El paso de Andrea Doria al bando imperial en 1528 coincide con la puesta en marcha de una reforma constitucional en la república que tenía por objeto facilitar la estabilidad política y superar, así, la constante ingerencia extranjera en la política interior genovesa derivada de los anteriores conflictos de facciones. El acuerdo con la Monarquía y el apoyo militar ofrecido por los Habsburgo permitieron garantizar dicho proceso de oligarquización a cambio de la colaboración naval y de las ventajas estratégicas brindadas por la república. Una relación simbiótica que estuvo a punto de resquebrajarse a causa de la

i1 RAGGIO, O.: Faide e parentele. Lo stato genovese visto dalla Fontanabuona, Turín, 1990; GRENDI, E., Il Cervo e la repubblica. Il modello ligure di antico regime, Turín, 1993.

12 PACINI, A.: «La repubblica di Genova vista da Simancas» Società e storia, 67, (1995), pp. 141-154. Sobre estas cuestiones véase también el interesante balance de BITOSSI, C.: «Genova, Spagna e Mediterraneo nel secondo cinquecento: bilanci e prospettive», en Anatra, Bruno y Manconi, Francesco, (eds.), Sardegna, Spagna e Stati italiani nell età di Filippo II, Cagliari, 1999, PP. 163188. No obstante, conviene señalar que los trabajos de Grendi conjugan una perspectiva en la que se enlaza su profundo conocimiento de la estructura social de la república de Génova con el contexto económico internacional pero sin perder de vista el papel central de los individuos y de sus redes de relaciones personales. Los artículos recopilados en GRENDI, E.: La repubblica aristocratica dei genovesi. Politica, carità e commercio fra Cinque e Seicento, Bolonia, 1987, y su magnífico trabajo sobre la familia Balbi constituyen dos contribuciones fundamentales para el estudio de la república, GRENDI, E.: I Balbi. Una famiglia genovese fra Spagna e Impero, Turín, 1997.

13 PACINI, A.: I presuposti politici del 'secolo dei Genovesi': la Riforma del 1528, Génova, 1990. 
revuelta de 1575 encabezada por los sectores menos proclives a la causa española. El autor aporta una nueva perspectiva al clásico estudio de Savelli ${ }^{14}$ al situar el conflicto en el punto de mira de la Monarquía Hispánica y de las medidas emprendidas por Madrid para evitar un posible cambio de alianzas por parte de los rebeldes. La rica información procedente del Consejo de Estado permite observar la complejidad en el proceso de toma de decisiones y las distintas posturas adoptadas en la Corte para hacer frente al problema. El papel de mediador ejercido por el embajador español en Génova y las, en apariencia, contradictorias medidas auspiciadas desde Madrid ayudaron, aunque fuera de modo involuntario, a restablecer la unidad del patriciado genovés gracias a la publicación de las Leges Novae de 1576 que dotaban a la república de una constitución que iba a perdurar hasta 1797 en un clima de inusitada estabilidad.

Los dramáticos acontecimientos de 1575-1576 constituyen el punto de partida del estudio de Thomas Kirk, uno de los principales especialistas en historia naval de la república ${ }^{15}$. En su artículo se aborda el periodo de mayor colaboración entre ambos socios y las características generales de dicha alianza hasta la década de 1620 cuando se empezaron a poner en cuestión, por ambas partes, los beneficios del acuerdo. El medio general de 1577 había permitido abrir el acceso a los préstamos a las principales familias de la nobleza nueva, lo que facilitó la posición de casi monopolio ejercida por los banqueros de la república sobre las finanzas de la corona. Salvo algunas disputas menores relativas al control español del enclave de Finale en la costa ligur ${ }^{16} \mathrm{o}$ a cuestiones de carácter simbólico como el problema de los saludos navales, que se nos describen a la luz de documentación genovesa pero también de los archivos de Venecia y Florencia, la armonía fue la tónica dominante hasta la bancarrota de 1627 sobre la que Kirk mantiene una posición divergente a la ofrecida por Álvarez Nogal.

Este panorama general en torno a la evolución de las relaciones hispanogenovesa se cierra con nuestra contribución sobre el proceso de distanciamiento entre ambos aliados iniciado con el estallido de la guerra de Mantua y con la paralela suspensión de pagos de 1627 . La crisis de las ferias cambiarias del norte de Italia y las dificultades de la Monarquía para hacer frente a una situación de guerra total terminaron por erosionar los fundamentos del acuerdo y facilitaron el acceso al poder del denominado partido republiquista que apostaba por una posición de neutralidad activa mediante un programa de rearme naval

14 SAVELLI, R: La repubblica oligarchica. Legislazione, istituzioni e ceti a Genova nel '500, Milán, 1981.

15 Estamos a la espera de la publicación de su libro sobre la política naval genovesa entre 1559 y 1680. Una buena síntesis sobre estas cuestiones en KIRK, T.: "A Little Country in a World of Empires: Genoese Attempts to Penetrate the Maritime Trading Empires in the Seventeenth Century», The Journal of European Economic History, 25, no. 2 (1996), pp. 407-421

16 Sobre el conflicto de Finale y las relaciones de Génova con el Imperio véanse los trabajos de Edelmayer, F.: Maximilian II, Philip II und Reichsitalien. Die Auseinandersetzungen um das Reichsleben Finále in Ligurien, Stuttgart, 1988 y "Genova e l'Impero nel Cinquecento», Atti della Società Ligure di Storia Patria, Nuova Serie, XLI/II, (2002), pp. 123-134.

Hispania, LXV/1, núm. 219 (2005) 9-20 
y el establecimiento de acuerdos alternativos con otras potencias. La tensión alcanzó su máxima expresión en 1654 cuando fueron confiscados los bienes genoveses en los territorios italianos de la Monarquía. A partir de ese momento, la derrota militar española y la política expansionista llevada a cabo por Luis XIV facilitaron un nuevo intento de acercamiento que no fue suficiente para evitar el masivo bombardeo de Génova por la flota francesa en 1684. El sistema imperial hispano-genovés cedía el paso a otros modelos dinásticos más centralizados y homogéneos con la suficiente capacidad para no tener que depender de los recursos financieros y navales ofrecidos hasta entonces por las repúblicas mercantiles.

El protagonismo que le hemos concedido a las transformaciones políticas y sociales derivadas de la mutua relación de dependencia hispano-genovesa y a la vertiente financiera del acuerdo no significa que no le demos importancia a otros ámbitos de análisis sobre los que se han realizado interesantes aportaciones y en torno a los que nos gustaría realizar unas reflexiones finales. Nuestra intención es la poner el acento sobre determinadas cuestiones que no han. sido abordadas con el necesario rigor o que pueden abrir posibilidades de análisis para futuras investigaciones.

El imponente arraigo de las comunidades genovesas en las principales ciudades y plazas comerciales de la Monarquía y su extensa y bien coordinada red de socios y parientes, así como los mecanismos de protección corporativa puestos en marcha para salvaguardar sus intereses de grupo (hospitales, cofradías, capillas, consulados), no han sido suficientemente estudiados a pesar de las abundantes fuentes disponibles. Por supuesto que contamos con trabajos bien documentados como los efectuados por Ruth Pike, Vila Vilar, Ladero Quesada, Collado Villalta o Álvarez Nogal para el caso de Sevilla ${ }^{17}$; Musi, Muto, Colapietra, Mantelli, Calabria, Spagnoletti, Zanini o Brancacio para Nápoles ${ }^{18}$; Tra-

17 PIKE, R.: Enterprise and Adventure: the Genoese in Seville and the Opening of the New World, Cornell, 1966; Collado Villalta, P.: «La nación genovesa en la Sevilla de la Carrera de Indias: declive mercantil y pérdida de la autonomía consular» en Presencia italiana en Andalucía, siglos XIV-XVII. Actas del I Coloquio Hispano-italiano, Sevilla, 1985, pp. 53-114; VILA VILAR, E.: Los Corzo y los Mañara. Tipos y arquetipos del mercader con América, Sevilla, 1991 o, de la misma autora, "Participación de capitales italianos en las rentas de Sevilla en el siglo XVI» en Presenza italiana nell'Andalusia del basso medioevo, Bolonia, 1990, pp. 85-102; LADERO QuESADA, M.A.: «I genovesi a Siviglia e nella sua regione: elementi di permanenza e di radicamento (secoli XIII-XVI)» en Del Treppo, M., Sistema di rapporti internazionali ed élites economiche in Europa (secoli XII-XVII), Nápoles, 1994, pp. 211-230; Álvarez Nogal, C.: Sevilla y la Monarquia Hispánica en el siglo XVII, Sevilla, 2000.

18 COLAPIETRA, R.: «I genovesi a Napoli nel primo Cinquecento», Storia e politica, 6-7(1968) págs. 386-419 así como, del mismo autor, «Le rendite genovese in Terra di Bari alla fine del Seicento» Rivista Storica del Mezzogiomo, 2, (1967) pp. 153-167 y «Genovesi in Calabria nel Cinque e Seicento", Rivista storica calabrese, 2, (1981) pp. 15-89; MANTELLI, R.: Burocrazia e finanze pubbliche nel Regno di Napoli, Nápoles, 1981 CALABRIA, A.: «Finanzieri genovesi nel Regno di Napoli nel Cinquecento", Rivista Storica Italiana, 101, (1989), pp. 578-613 y, del mismo autor, The Cost of Empire. The Finances of the Kingdom of Naples in the Time of the Spanish Rule, Cambridge, 1991; MUSI, A.: Mezzogiorno spagnolo. La via napoletana allo stato moderno, Nápoles, 1991; MUTO, G.: "Cittadini e

Hispania, LXV/1, núm. 219 (2005) 9-20 
selli, Aymard y Giuffrida para Sicilia ${ }^{19}$ u otras aportaciones puntuales sobre los Países Bajos ${ }^{20}$, Cartagena ${ }^{21}$, Cádiz ${ }^{22}$ o Madrid ${ }^{23}$. De todos modos aún queda mucho trabajo por hacer en este terreno y es necesario ofrecer una visión que trascienda de los meros estudios locales y que ponga en conexión a estas comunidades con otros nudos de dicha red, en especial con Génova y con Madrid. Además convendría abrir el campo de análisis a los sectores más modestos de las comunidades genovesas, artesanos, marineros o pequeños comerciantes, cuya presencia fue mayor de la que se suele apuntar.

De esta manera lograríamos entender, de un modo más matizado, el impacto y las transformaciones mutuas, derivados del establecimiento de tan estrechas e intensas relaciones, en el seno de las sociedades de acogida y de las comunidades genovesas. Los hombres de negocios procedentes de la república de Génova gozaron de una serie de ventajas comparativas en relación a sus rivales portugueses, ingleses $u$ holandeses que les permitieron promocionarse en el interior de los gobiernos locales y acceder con mayor facilidad a los beneficios derivados de la política de patronazgo regio. Dejando a un lado su amplio respaldo financiero y su extensa red de contactos, es necesario recordar que los más insignes miembros de las numerosas comunidades genovesas arraigadas en los territorios de la Monarquía Hispánica estaban en posesión de un lustre aristocrático del que carecía buena parte de sus contrincantes, debido a su pertenencia a alguno de los alberghi que mantenían un control exclusivo sobre los cargos públicos de la república. Las sucesivas bancarrotas facilitaron la promoción social de estos grupos que, a cam-

'forestieri' nel regno di Napoli: note sulla presenza genovese nella capitale tra Cinque e Seicento» en Del Treppo, M. (ed.), Sisteme di rapporti internazionali...cit., pp. 164-178; SPAGNOLETTI, A.: Principi italiani e Spagna nell'età barroca, Milán, 1996; ZANINI, A.: "Gio Tomasso Invrea, un finanziere genovese nella Napoli del Seicento", Atti della Società Ligure di Storia Patria, XLI, 2, (2001), pp. 49104; BRANCACCIO, G.: «Nazione genovese». Consoli e colonia nella Napoli moderna, Nápoles, 2001.

19 Trasselli, C.: «Los genoveses en Sicilia y en Calabria desde el reinado de Carlos V hasta la guerra de los Treinta años», en A. Otazu (ed.) Dinero y Crédito (siglos XVI al XIX), Madrid, 1978, pp. 197-205; AYMARD, M.: «I genovesi e la Sicilia durante la guerra dei trent' anni. Bilancio di una lunga crisi finanziaria», Rivista Storica Italiana, 84, (1972), pp. 275-332; GIUFFRIDA, R.: «Aspetti dell'attività finanziaria genovese nella Sicilia spagnola» en Genova e i genovesi a Palermo, Palermo, pp. 61-84.

20 JANSSENS, P. y DeNEWETH, C.: «Les relations entre Gênes et les Pays-Bas espagnols (15551702). Etat de la recherche ", en Belvderi, R. (ed.), Atti del IV congresso internazionale di studi storici. Rapporti Genova-Mediterraneo-Atlantico nell'Età Moderna, Génova, 1990, pp. 241-257.

21 MONTONJO MONTOjO, V.: «Crecimiento mercantil y desarrollo corporativo en España: los consulados extraterritoriales de extranjeros (ss. XVI-XVII)», Anuario de Historia del Derecho Español, (1992), pp. 47-66.

22 SANCHO DE Sopranis, H., Los genoveses en Cádiz antes de 1600, Larache, 1939. Un buen comentario sobre las ricas memorias de Raymundo Lantéry, que ponen de manifiesto el poder alcanzado por los genoveses en dicha ciudad, es el de BusTOS RoDríGuEZ, M.: Un comerciante saboyano en el Cádiz de Carlos II, Cádiz, 1983.

23 SANZ AYÁN, C.: «Bajo el signo de Júpiter: negocios y hombres de negocios en el Madrid del seiscientos», en Morán, M. y García García, B. (eds.), El Madrid de Velázquez y Calderón. Villa y Corte en el siglo XVII.Vol. I. Estudios bistóricos, Madrid, 2001, pp. 61-79.

Hispania, LXV/1, núm. 219 (2005) 9-20 
bio, obtuvieron un acceso privilegiado a las más lucrativas rentas de la corona y lograron controlar los mejores juros y adquirir con facilidad hábitos de las órdenes militares o determinados títulos nobiliarios. Su capacidad como administradores y su conocimiento de los complejos mecanismos de la hacienda regia facilitarían también su acceso a determinados cargos de relevancia en el gobierno de la Monarquía. Algo semejante se produjo en el ámbito municipal o en las gobernaciones y virreinatos bajo la jurisdicción del monarca Católico. La facilidad con la que los principales miembros del patriciado de la república enlazaron con la elite local en Castilla, Nápoles, Sicilia o Milán explica que, hasta el momento, se haya puesto el acento en el proceso de ennoblecimiento y en el abandono de las actividades mercantiles por parte de los comerciantes genoveses. Ahora bien, es necesario recordar que el capital mercantil genovés dotó de energías renovadas al sistema señorial dominante y permitió, en un proceso de mutua transformación, introducir mejoras sustanciales en la gestión de los patrimonios nobiliarios y facilitar el acceso a una serie de productos de lujo fundamentales para reforzar el estatus social de las elites.

En este sentido convendría observar con más calma la influencia determinante ejercida por los modelos arquitectónicos y las formas estéticas procedentes de Génova en la creación de un lenguaje cultural de fuertes tintes cosmopolitas y en la difusión de unos modelos que fueron imitados en el resto de Europa hasta finales del siglo XVII. Lejos de contentarse con reproducir el modo de vida de las elites locales, los genoveses introdujeron nuevas pautas de consumo y marcaron el camino a seguir en la construcción de sus palacios además de facilitar la propagación de determinadas formas refinadas de comportamiento. Además de los trabajos de Doria o de Poleggi sobre la renovación urbanística emprendida por el patriciado genovés o de Magnani sobre la tipología de los jardines de sus suntuosas residencias ${ }^{24}$, contamos con una reciente contribución coordinada por Boccardo, Colomer y Di Fabio sobre las transferencias culturales hispano-genovesas que parece haber abierto un amplio abanico de posibilidades para futuras investigaciones ${ }^{25}$.

Un buen método para obtener un conocimiento más vivo de la manera en la que se tradujeron estas relaciones de mutua dependencia en una serie de realidades concretas, consiste en el estudio de determinados casos particulares. En este senti-

24 DORIA, G.: «Investimenti della nobiltà genovese nell'edilizia di prestigio (1530-1630)» en Doria, Giorgio, Nobiltà e investimenti a Genova in Età Moderna, Génova, 1995, págs. 235-285; PoLEGGI, E.: Strada Nuova. Una lottizzazione nella Genova del Cinquecento, Génova, 1972; MAGNANI, L.: "The Rise and Fall of Gardens in the Republic of Genoa, 1528-1797» en Conan, M. (ed.), Bourgeois and Aristocratic Cultural Encounters in Garden Art, 1550-1850, Washington, 2002, pp. 43-76.

25 Se incluyen trabajos sobre un buen número de aspectos relacionadas con las formas de transferencia de objetos suntuarios, esculturas y pinturas con atención especial al estudio del mercado artístico o de la iconografía. No se dejan, sin embargo, de lado cuestiones referentes a la representación simbólica del poder o a la percepción mutua entre ambas realidades, BOCCARDO, P., COlOMER, J.L. y Di FABIO, C. (Eds.): Genova e Spagna. Opere, artisti, comittenti, collezionisti, Milán, 2002.

Hispania, LXV/1, núm. 219 (2005) 9-20 
do, la biografía realizada por Grendi sobre la familia $\mathrm{Balbi}^{26}$ o los aportes tangenciales de Tagliaferro sobre los Brignole Sale ${ }^{27}$ constituyen un posible modelo de análisis para el que contamos con la existencia de ricos archivos privados. En la actualidad, Álvarez Nogal está trabajando en una biografía sobre el factor general Bartolomé Spinola que le permitirá contextualizar su conocimiento sobre las actividades empresariales de los grandes asentistas de la corona en su red de relaciones personales. Algo semejante debería hacerse con aquellas familias genovesas que, como los Doria o los Spinola, lograron alcanzar la grandeza de España y sobre las que existe abundante material que nos permitiría trazar la trayectoria de algunos de los principales hombres de estado y militares al servicio del rey Católico ${ }^{28}$.

Por último, y en concordancia con la importancia de Génova para el buen funcionamiento del sistema de comunicaciones entre los dispersos territorios de la Monarquía y para asegurar la defensa en el Mediterráneo occidental, resulta incomprensible que, a pesar de la riquísima documentación existente, no se haya realizado aún un estudio sistemático sobre las escuadras de galeras genovesas. Más que como un negocio lucrativo, el asiento de galeras constituía un instrumento de negociación política con la corona para lograr nuevas prebendas o mejores condiciones en la renovación de los préstamos y para obtener una serie de inmunidades que facilitaban el contrabando de mercancías y de metales preciosos gracias a los permisos que se les concedía par transportar las inmensas partidas de numerario remitidas a Italia.

Hemos tratado tan sólo de apuntar algunas nuevas posibilidades de análisis que, por supuesto, están lejos de cubrir las infinitas posibilidades que se derivan del estudio de la república de Génova como una adecuado marco desde el que obtener una mejor comprensión sobre la evolución del entramado imperial hispánico. Si, como decía Braudel, «l'automne de Gênes fut l'automne de l'Espagne» ${ }^{29}$, resulta incontestable que la ruptura de los lazos privilegiados que ligaban a la república con la Monarquía a finales del siglo XVII redujo de modo dramático las posibilidades de promoción de sus elites y provocó un desplazamiento de Génova de la posición central que había jugado hasta entonces en el escenario internacional.

26 GRENDI, E.: I Balbi. Una famiglia genovese...cit.

27 TAgliaferro, L.: La magnificenza privata. Gioie, quadri, e altri mobili della famiglia Brignole Sale, Génova, 1995.

28 Los trabajos existentes sobre Ambrogio Spinola están lejos de cubrir las amplias posibilidades que ofrece el personaje para entender el funcionamiento de la Monarquía y sus fuertes vínculos con Génova, RODRíGUEZ VILLA, A.: Ambrosio Spinola, primer marqués de los Balbases. Ensayo biográfico, Madrid, 1904; BRANTS, V.: «Ambroise Spinola (1569-1630), généralissime des armes de Flandres», Revue Générale Belge, 1 (1915), págs. 172-202 y LEFEVRE, J.: Spinola et la Belgique, Bruselas, 1947. Aunque pueda parecer extraño, no disponemos todavía de un estudio completo sobre la figura de Andrea Doria, salvo las ya reseñadas aportaciones de Pacini o el trabajo de GrENDI, E.: «Andrea Doria uomo del Rinascimento» en Grendi, E., La repubblica aristocratica....cit., pp. 139-172.

29 BRAUDEL, F.: «Le siècle des génois s'acheve-t-il en 1627?» en Braudel, F., Au tour de la Méditerrannée, París, 1996, p. 446. 